\title{
Post-traumatic Delayed Peripheral Facial Palsy
}

\author{
(1) Leyla Kansu
}

Başkent University Faculty of Medicine, Department of Ear, Nose and Throat, Ankara, Turkey

\begin{abstract}
Peripheral facial palsy in children is very rare in comparison to adults. The most common cause is idiopathic. Another rare cause of peripheral facial palsy in children is trauma. It occurs after head trauma, mostly due to temporal bone fracture as an early onset paralysis after trauma. Early onset facial palsy is usually due to direct damage to the facial nerve whereas there is some controversy about the etiology of late onset facial palsy. In this article, a child patient whose peripheral facial palsy developed six days after a head injury is presented, and the etiopathogenesis and the treatment of delayed traumatic facial palsy after temporal bone fracture is discussed.

Keywords: Head trauma, facial palsy, child, etiopathogenesis, treatment
\end{abstract}

\section{Introduction}

Facial paralysis (FP) in children is an uncommon condition, although it is common in adults. Its estimated annual incidence is thought to be approximately $2.7 / 100.000$ in children younger than 10 years (1). In spite of this, there are several described causes of facial nerve paralysis in children, in approximately $40-75 \%$ of cases, the cause of unilateral FP is still unknown and it remains idiopathic (2). The most common known causes of FP are Herpes Simplex Type I, varicella zoster virus, Ebstein-Barr virus, hemophilus influenza, tuberculosis, Lyme disease, adenovirus, rhinovirus, acute and/or chronic otitis media, ear infection with cholesteatoma, mastoiditis, vasculitis, inflammatory disease such as Henoch-Schönlein purpura, Kawasaki syndrome and neoplastic tumors $(1,2)$. FP due to fracture of the temporal bone after head trauma is not common. It accounts for $1.5-5 \%$ of the causes of FP in children, and is mostly seen immediately following a head injury (3-5). The incidence of delayed facial palsy after head injury was found to be approximately $0.6-2.2 \%$ (6). In this article, a child patient whose peripheral facial palsy developed six days after head injury is presented, and the etiopathogenesis and the treatment of delayed traumatic facial palsy following a temporal bone fracture is discussed.

\section{Case Report}

A-8-year-old girl with right peripheral FP was sent to our ear, nose and throat (ENT) clinic from the emergency service. In her medical history taken from her family, she had fallen from a bicycle 6 days earlier. On the day of the accident, she arrived at the emergency service, a computed brain tomography was taken and it appeared normal. Her physical and neurological examinations revealed no abnormalities and she was discharged. Five days after her head trauma her family noticed immobilization of the right side of her mouth and the inability to close her right eye, and they applied to the emergency department. There was no complaint such as ear pain, bleeding from the ear, hearing loss or vertigo. On otological examination, the right ear drum was intact, but there was hemotympanum in the anterior zone of her right ear drum. Her neurologic examination revealed the development 
of a House-Brackmann Grade III-IV peripheral FP on the right side (Figure 1). The other examinations were normal. In the patient's hearing test, an average $28 \mathrm{~dB}$ conductive hearing loss was found in the right ear (Figure 2). High resolution temporal bone computed tomography (HRTBCT) of the patient was taken, which revealed hemorrhage and edema in the right middle ear cavity and a mastoid cellular and longitudinal temporal bone fracture without bone chain damage (Figure 3a, 3b). It was seen that the fracture line crossed at the geniculate ganglion of the facial nerve (Figure 4). Corticosteroids were administered to the patient at a dosage of $1 \mathrm{mg} / \mathrm{kg} / \mathrm{d}$ and it was decreased progressively and stopped after 18 days. By the end of one week, FP had begun to improve. She was examined after one month; her FP and hearing deficit were resolved completely. Written informed consent was obtained from the patient's parents.

\section{Discussion}

The trauma patient constitutes most of the patients who apply to the emergency department (7). Traumatic head injury is a smaller group of these patients. Basal skull fractures account for $21 \%$ of all skull fractures. In approximately $7-8 \%$ of these individuals, temporal bone fractures occur. In the pediatric population, the incidence of temporal bone fracture

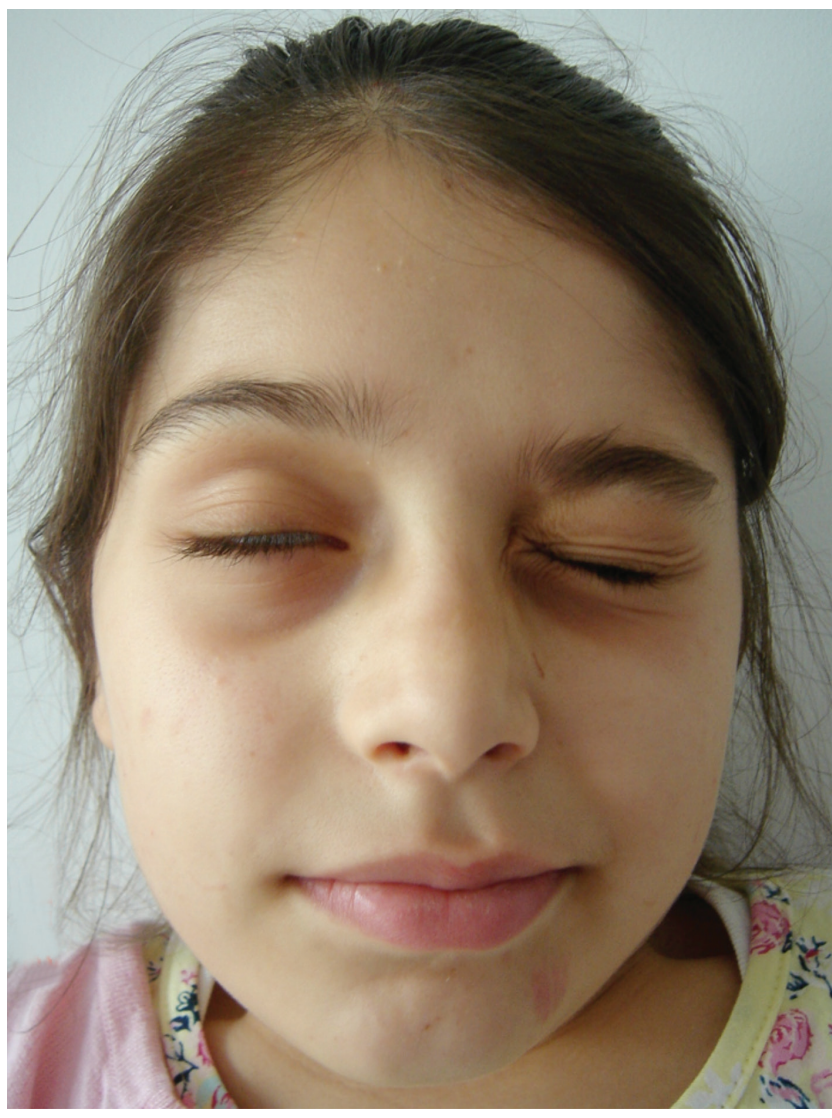

Figure 1. The patient with right peripheral facial palsy

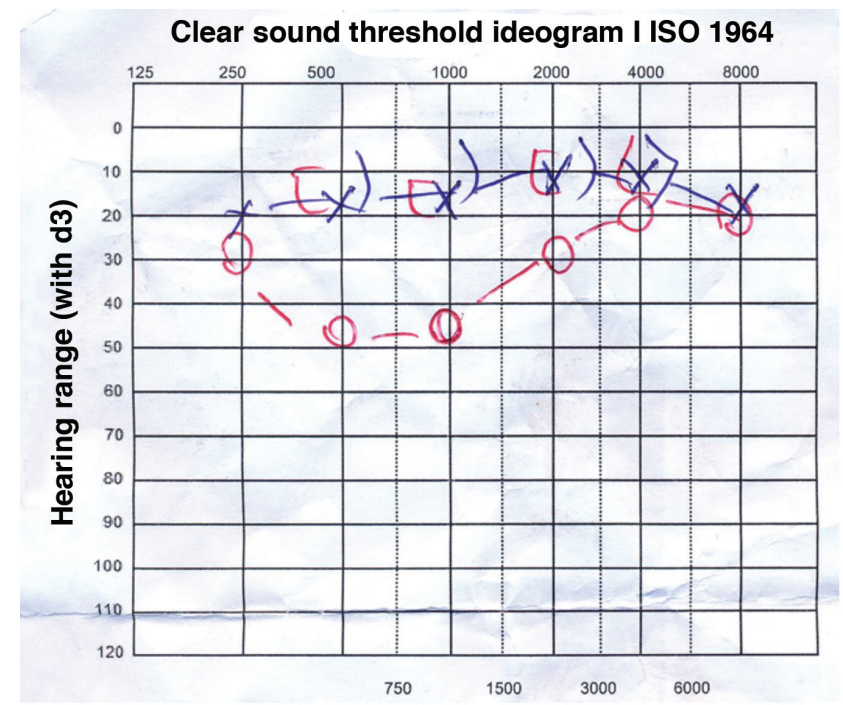

Figure 2. The hearing test of the patient after trauma

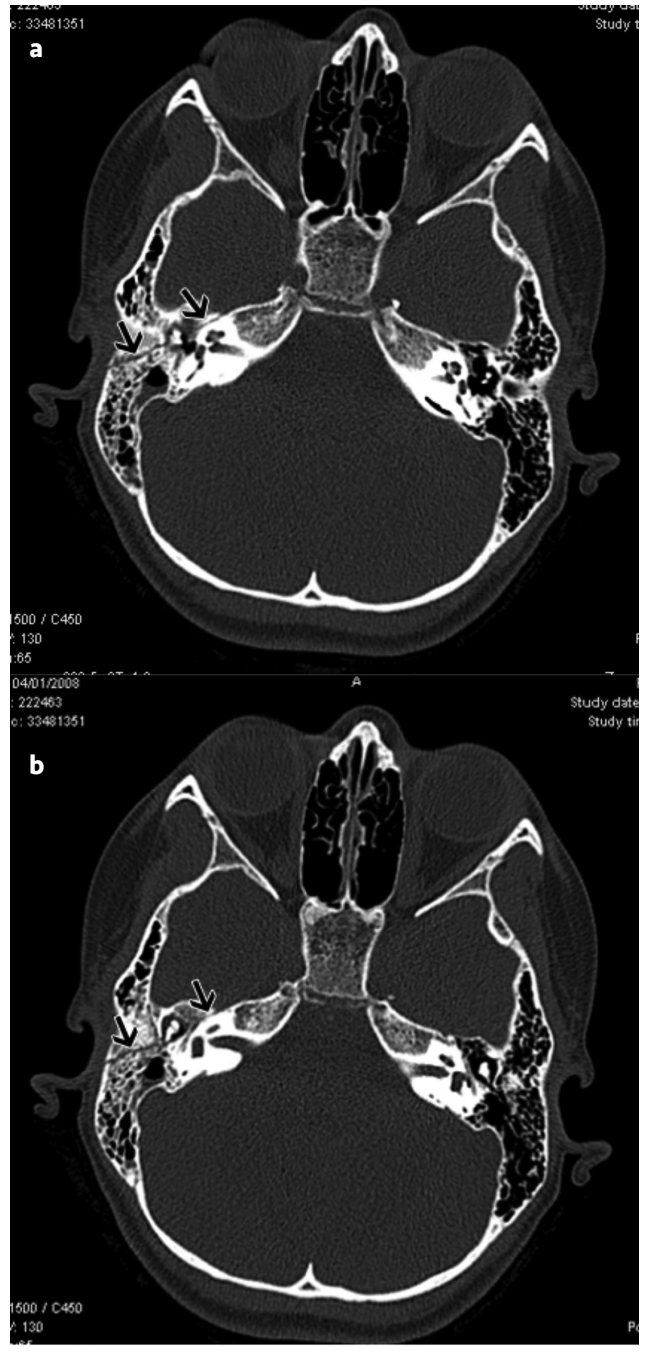

Figure $3 \mathbf{a}, \mathbf{3 b}$. The image of coronal section high resolution computed tomography of the patient 


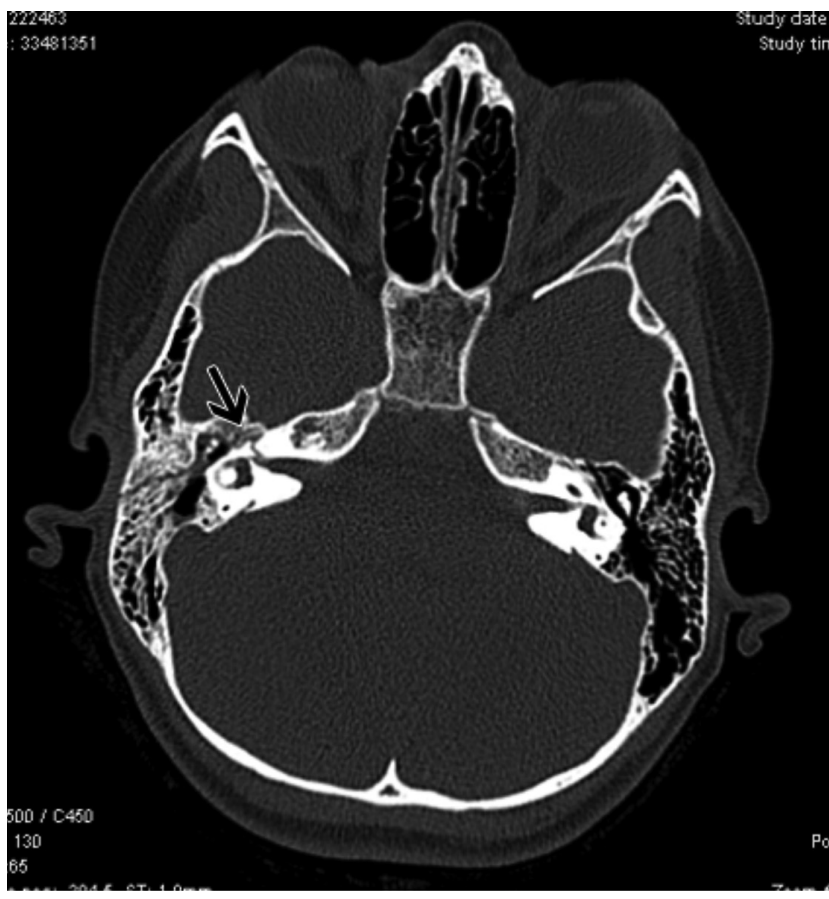

Figure 4. The fracture line which crossed the geniculate ganglion section of facial nerve (arrow)

is not common (7). Temporal bone fractures are classified as longitudinal, transverse and mixed with respect to the long axis of the petrous bone: in longitudinal fractures; the fracture line is parallel to the long axis of the petrous bone, in transverse fractures; the fracture line crosses the long axis of the petrous bone, in mixed fractures; there are both fracture lines (8). Up to $80 \%$ of all temporal bone fractures are longitudinal as was seen in our patient. Longitudinal fractures very often pass through the external auditory canal, and FP occurs in $10-20 \%$ of these cases $(6,9)$. Transverse fractures are uncommon and account for only $15 \%$ of temporal bone fractures. In these patients, trauma usually occurs in the occipital and frontal region. Facial nerve paralysis occurs in $50 \%$ of transvers fractures, and the paralysis is likely to be immediate in onset. Mixed type fractures are very rare, they account for $5 \%$ of all temporal bone fractures (9). The severity of head trauma affects both the severity of temporal bone fracture and the development of FP. Presently, the use of seat belts and more recently airbags has dramatically decreased the incidence of temporal bone fractures (10).

In the immediate onset of facial nerve palsy after trauma, the nerve is either completely lacerated or contused at the fracture site $(4,6)$. The delayed presentation of FP is seen typically 1-10 days after injury. In our patient, FP developed over 6 days. There are different ratios about post-traumatic delayed facial palsy in different studies. Turner found a $2.2 \%$ ratio in their study while Puvanendran et al. (6) found the ratio to be $0.3 \%$. To our knowledge, there is no study in the literature about this issue in children. The pathophysiology of post-traumatic delayed facial palsy is not clearly known. Some theories have been suggested about this issue: in the facial canal, the area occupied by the facial nerve is only $30-50 \%$ of the cross-sectional area of the canal. The remainder of the facial canal is occupied by blood vessels with connective tissue loosely arranged around the nerve. Delayed facial palsy is possibly the result of bleeding into the facial canal. An increasing size of a hematoma in the limited non-expanding bony tube could press on the facial nerve. If the pressure were of a mild degree, there would only be a neuropraxia, or conductive block due to segmental demyelination. If the damage were more severe, there could be axonal damage with denervation (6). The other theory is that ultimately the blood supply of facial nerve is cut off due to the trauma and this causes ischemic damage to the nerve. Some authors postulate a similar mechanism for this facial weakness as in Bell's palsy with a possible inflammatory reaction in and around the nerve, or a swelling of the nerve in the canal which could lead to ischemia. The vascular damage such as delayed arterial spasm, arterial or venous thrombosis, external compression from bony fragment or soft tissue edema are other etiological causes $(4,5,9)$. The symptoms and clinical findings in temporal bone fractures change depending on whether there is a longitudinal or transverse fracture line. Longitudinal fractures very often pass through the external auditory canal, and usually tear the tympanic membrane producing bleeding from the external auditory area and leading to conductive hearing loss. Transverse fractures cause vertigo, hemotympanum and sensorineural hearing loss (6). In this condition, otological examination should be carried out and the patient should be referred to an ENT specialist. HRTBCT imaging should be taken to assess facial nerve damage. In the treatment of post-traumatic FP, it is important to identify patients with immediate or delayed onset facial palsy. The treatment protocol should as below (10):

In cases of immediate FP after trauma and electrophysiologically severe with a clear cut fracture line on the fallopian canal on high-resolution CT (HRCT), surgery is performed as soon as possible; depending on the patient's neurologic status. Facial nerve repair is achieved by reanastomosis of the severed ends or, in cases with significant loss of nerve tissue, cable grafts using the great auricular nerve, the sural nerve or the cervical plexus as donor sites (4).

- In cases of immediate FP in the absence of a visible fracture line on $\mathrm{HRCT}$, a medical treatment with steroids is given. The patient is followed with electrophysiological tests over a period of 3-6 months. Crushing or stretching injuries without cutting the nerve cause interruptions in nerve electric conduction. Stretching injuries heal more slowly than crushing injuries and they differ in range and degree. Surgery may be performed if there is no recovery in terms of both 
electrophysiological tests and clinical tests 6 months after trauma $(9,10)$.

- In cases of FP a few days after trauma, even if a visible fracture line is present on $\mathrm{HRCT}$, the patient is followed with medical treatment. Steroids are given to reduce inflammation and edema in the nerve. Corticosteroid treatment initiated at a dosage of $1 \mathrm{mg} / \mathrm{kg} / \mathrm{d}$ is given for 3 weeks $(1,7)$.

The time of surgery time is controversial. If surgery is performed as early as possible, the functional and aesthetic results are more likely to be better than if the surgery is delayed. If the nerve was cut, the repair of it should be completed within 72 hours from the onset of the trauma (2). Prognosis of post-traumatic FP depends on the time of onset of the paralysis, the degree of paralysis and the site of the injury. Delayed post-traumatic FP usually has a good prognosis. Most patients of post-traumatic facial injuries recover with conservative treatment. Surgery and reanimation are rarely required (9). As a consequence; although the incidence of post-traumatic FP in children is very low, as soon as this issue is recognised, it must be immediately treated. Emergency service doctors should be alert to all issues concerning children with head-injuries. When a child with post-traumatic FP comes to an emergency department, the emergency department physician should take a detailed history and perform a detailed physical examination, arrange a cranial CT scan and refer the patient to a brain surgeon and ENT specialist to establish the most appropriate treatment as quickly as possible.

\section{Ethics}

Informed Consent: Written informed consent was obtained from the patient's parents.
Peer-review: Externally peer-reviewed.

Financial Disclosure: The author declared that this study received no financial support.

\section{References}

1. Kansu L, Yazıcı N, Ergün T. Bilateral simultaneous Facial Palsy in a pediatric patient. Mediterr J Otol 2008;4:230-3.

2. Ciorba A, Corazzi V, Conz V, Bianchini C, Aimoni C. Facial nerve paralysis in children. World J Clin Cases 2015;3:973-9.

3. Evans AK, Licameli G, Brietzke S, Whittemore K, Kenna M. Pediatric facial nerve paralysis: patients, management and outcomes. Int J Pediatr Otorhinolaryngol 2005; 69:1521-8.

4. Baumann BM, Jarecki J. Posttraumatic delayed facial nerve palsy. Am J Emerg Med 2008;26:115.e1-2.

5. Khangwal M, Solanki R, Bali A, et al. Delayed post traumatic facial nerve palsy on contra lateral side of isolated mandibular fracture: a rare case report. Int J Dent Health Sci 2014;1:89-98.

6. Puvanendran K, Vitharana M, Wong PK. Delayed facial palsy after head injury. I Neurol Neurosurg Psychiatry 1977;40:342-50.

7. Napoli AM, Panagos P. Delayed presentation of traumatic facial nerve (CN VII) paralysis. J Emerg Med 2005;29:421-4.

8. Wexler S, Poletto E, Chennupati SK. Pediatric temporal bone fractures: A 10-year experience. Pediatr Emerg Care 2017;33:745-7.

9. Turel KE, Sharma NK, Verghese I, Desai S. Post traumatic facial paralysis treatment options and strategies. Indian । Neurotrauma 2005;2:33-4.

10. Darrouzet V, Duclos JY, Liguoro D, el al. Management of facial paralysis resulting from temporal bone fractures: Our experience in 115 cases. Otolaryngol Head Neck Surg 2001;125:77-84.

11. Turner JWA. Facial palsy in closed head injuries the prognosis. Lancet 1944;243:756-7. 